\title{
¿Es la cárcel violenta en sí misma? Violencia y legitimación de la dinámica institucional en el Centro de Formación Juvenil Zurquí, Heredia, Costa Rica
}

\author{
Mario Araya-Pérez \\ Programa Agenda Joven, Universidad Estatal a Distancia, San José, Costa Rica \\ solem.araya@gmail.com
}

\begin{abstract}
Resumen: El siguiente artículo tiene como objetivo la comprensión de la violencia en el Centro de Formación Juvenil Zurquí (CFJZ), como un emergente del conjunto de relaciones establecido por los diferentes actores involucrados en el funcionamiento diario de este; los cuales se encuentran mutuamente imbricados en el proceso de constitución de un entorno carcelario en el que cada uno ocupe un lugar y adquiera un sentido. La investigación se desarrolló a partir de una metodología cualitativa que consistió en un trabajo de campo directo en los espacios de convivencia de la población, por un periodo de ocho meses durante el 2015, el cual estuvo dividido en tres fases. Se logró determinar la participación de los diferentes actores en el ejercicio de diversas manifestaciones de violencia con miras a la ficcionalización de la persona en el entorno carcelario, el cual posibilita mostrar una cierta regularidad en los valores, actitudes y comportamientos para mantenerse encajados en la dinámica social y, por lo tanto, asegurar su supervivencia tanto a nivel material como simbólico en dicho contexto. Se llega a la conclusión de que la producción y reproducción de la violencia es el proceso mediante el cual se legitima la posición social de cada uno de los actores en los centros penales, al igual que la función de la institución penitenciaria en la sociedad en general.
\end{abstract}

Palabras clave: Agresividad; gregario; prisión; sociabilidad; privados de libertad.

Cuadernos de Antropología

Enero-Junio 2016, 26(1), 85-104

DOI: $10.15517 /$ cat.v26i1.25196

Recibido: 26-02-2016 / Aceptado: 17-05-2016 / Publicado: 28/06/2016

Revista del Laboratorio de Etnología María Eugenia Bozzoli Vargas

Escuela de Antropología, Universidad de Costa Rica

http://revistas.ucr.ac.cr/index.php/antropologia

ISSN 2215-356X

c) (i) (-) Cuadernos de Antropología está bajo una licencia Creative Commons Attribution-NonCommercial-ShareAlike 3.0 


\section{Is prison violent for itself? Violence and legitimation of institutional dynamics in the "Centro de For- mación Juvenil Zurquí", Heredia, Costa Rica}

Abstract: The following article aims to understand violence in the Zurquí Juvenile Training Centre as an emergent set of relations established by the different actors involved in the daily running of this. Actors, which are mutually involved in the process of establishing a prison environment where everyone has a place and sense. The research was developed from a qualitative methodology consisted of a direct fieldwork in the living spaces of the population, for a period of eight months during 2015 and divided into three phases. It was possible to determine the involvement of different actors in the exercise of various manifestations of violence towards the fictionalization of the person in the prison environment, which enables display a certain regularity in the values, attitudes and behaviors to remain embedded in social dynamics and therefore ensure their survival, both material and symbolic in that context. The research concludes that the production and reproduction of violence is the process by which the position of the actors involved is legitimized, while the function of the penitentiary institution in general society.

Keywords: Aggressiveness; gregarious; prison; sociability; deprived of their liberty.

\section{Introducción}

"La cárcel es violenta en sí misma" fue uno de los primeros señalamientos que me hacía una de las funcionarias del Centro de Formación Juvenil Zurquí1 (en adelante CFJZ) en mi primera visita para negociar el trabajo de campo en este centro penal juvenil (o cárcel de menores, como se le conoce popularmente). Para esta funcionaria no debía dejar de lado en mi investigación este aspecto fundamental. Pero, ¿qué significa que la cárcel sea violenta en sí misma? Una posible interpretación preliminar, parcial e insatisfactoria que se puede dar a esta expresión es que la cárcel somete a las personas privadas de libertad a condiciones y dinámicas de vida deplorable, llena de privaciones de todo tipo; una de las explicaciones más comunes relacionadas con la violencia en los centros penales (Michalski, 2015).

En Costa Rica se puede consultar una amplia cantidad de trabajos que han abordado el tema de los centros penales, se han realizado en estos o se ha trabajado con su población. La mayoría corresponde a investigaciones realizadas desde disciplinas como la Psicología, la Sociología y el Derecho, en temáticas sumamente variadas. Algunos de estos se han concentrado en el análisis de la ejecución y aplicación de las diferentes leyes a las personas privadas de libertad (Corrales y León, 2007; Rojas, 2013), así como en el cumplimiento de sus derechos (Fuentes, 1998) y los diferentes discursos en torno a la aplicación de las penas privativas de libertad (Arguedas, 1999; Rojas y Hernández, 2011); otras han analizado aspectos puntuales de la vida de las

1 El CFJZ se encuentra en el cantón de Santo Domingo de Heredia, sobre la ruta 32, carretera a Guápiles. Este centro fue puesto en operación en 1998, en las antiguas instalaciones del Centro de Formación Amparo Zeledón, cuyas instalaciones fueron construidas en 1978, cuando regía la Ley Tutelar de Menores. El centro fue diseñado para aplicar el modelo de atención de "comunidad terapéutica" en pequeñas unidades de socialización de mujeres menores de edad -entre 12 y menos de 18 años- llamadas "hogares". Estas se encontraban en el centro por la comisión de algún delito, cuasidelito o falta.

Cuadernos de Antropología 2016, 26(1), 85-104 / ISNN 2215-356X

http://revistas.ucr.ac.cr/index.php/antropologia 
personas privadas de libertad que los motivaron a la comisión de actos delictivos (Bustos, 2013; Ramírez, 2010; Vul 2009), la condición psicológica en la que se encuentra la población (Alpízar y Rodríguez, 2004) o bien considerado aspectos más estructurales de la sociedad a la que pertenecen quienes se encuentran en condición de encierro como aspectos determinantes en la comisión actos delictivos (Calderón, 2012).

Pese a esto, es poco frecuente encontrar trabajos en nuestro país que se aboquen a analizar las condiciones de vida en las cuales se encuentran las personas privadas de libertad, desde su experiencia diaria; cómo estas afectan la forma en la que se desenvuelven cotidianamente a lo interno de estos centros, así como el papel de los diferentes actores que en ella se encuentran, en aspectos tan relevantes como la violencia característica de este tipo de instituciones; como se procura hacer en este trabajo.

Este artículo analiza el papel de la violencia como posibilitador de las condiciones del entorno carcelario en el cual se desenvuelven los diversos actores involucrados en la dinámica diaria de la institución. Se propone que los individuos quienes son parte de dicha dinámica están mutuamente imbricados en una relación producto de la necesidad de asegurar la constitución de un entorno carcelario en el que cada uno de los actores ocupe un lugar y adquiera un sentido en la dinámica diaria que ahí se desarrolla. Ser parte de, ser reconocido como y pertenecer a este grupo social que se conforma de la multiplicidad de relaciones entre actores, cumpliendo los requerimientos específicos establecidos para cada uno de ellos, es lo que mueve a las personas a la (re) producción de las diversas manifestaciones en las cuales se concretiza la violencia en los centros penales (Zimbardo, 2015).

\section{Recolección de datos}

Este artículo es parte de un proceso de investigación más amplio que consistió en un trabajo de campo en profundidad con las personas jóvenes privadas de libertad del CFJZ, durante aproximadamente ocho meses, entre enero y agosto del 2015. La mayor parte del trabajo se efectuó con dos de las secciones en las cuales se encuentran personas de baja contención, quienes tienen una mayor libertad y baja custodia para su circulación por el centro.

El trabajo de campo se dividió en tres fases. Una primera fase mediante la observación participante consistió en la convivencia con la población varios días a la semana durante horarios de 8 de la mañana a 4 de la tarde. Con esto se buscó permitir un acercamiento gradual a la realidad y a las personas que son parte de la dinámica diaria del centro penal. Se logró ganar la confianza tanto de la población como de los funcionarios del centro y asegurar una guía de observación que posibilitó un registro más sistemático de datos. El segundo momento consistió en la aplicación de la guía de observación, en espacios y momentos relevantes de la convivencia de los individuos que son parte de los diferentes sectores que interactúan diariamente en el centro. La tercera face consistió en la aplicación de entrevistas en profundidad a un total de ocho personas privadas de libertad, para lo cual se empleó una guía de entrevista basada en la observación 
realizada hasta el momento. Además se aplicó una entrevista a una funcionaria de gran experiencia en el centro y se recolectaron otros materiales afines a la investigación.

\section{Violencia y sociabilidad}

Dar una definición de violencia requiere determinar a qué es a lo más básico que podemos llegar en cuanto a su origen, a qué está arraigado. En parte, las definiciones que no pueden distanciarse de las explicaciones basadas en la agresividad, como instinto básico del cual emerge la violencia, como si esta fuera su expresión socialmente visible, están relacionadas con este objetivo (Baños, 2005), entender qué es lo más básico, en la constitución del ser humano, que explica la violencia. La idea arraigada de que lo que mueve a las personas es la agresividad nos suele jugar en contra al momento de reflexionar sobre la violencia (Bauer, 2013). Cuando pensamos en ejemplos cotidianos con los cuales podamos ir anclando a la realidad los enunciados que desarrollamos en nuestras argumentaciones teóricas, constantemente recurrimos a aquellas situaciones donde las personas realizan un despliegue corporal en el que la fuerza física, la velocidad, la concentración y la tenacidad se hacen manifiestos en las dimensión física del cuerpo ${ }^{2}$; ya autoras como Arendt (2006) han señalado el peligro de este tipo de razonamientos.

A partir de las diferentes discusiones revisadas en los trabajos que abordan el tema de la violencia (Arendt, 2006; Baños, 2005; Blair, 2004; Cerbino, 2011; Chaves, 2011; Crespo, 2009; de Barcellos, 2010; Padrón, 2006; Sanmartín, 2002; Žižek, 2009; Zúñiga, 2012), parece cada vez más necesario dejar de lado la idea de la agresividad en las conceptualizaciones de la violencia, sobre todo aquellas que la consideran como un elemento instintivo del ser humano (Bauer, 2013).

Si bien algunos autores han intentado que la violencia y la agresividad sean diferenciadas a nivel conceptual, y que no se considere una relación lineal entre una y otra, es decir que a mayor agresividad mayor violencia (Baños, 2005), los problemas que estas siguen planteando son especialmente infructíferos en sus intentos de solución. Las propiedades atribuidas tanto a la agresividad como a la violencia tienden también a oscurecer la discusión sobre el concepto. La intencionalidad de daño, la búsqueda de beneficios propios en detrimento de los otros, la irracionalidad, o la separación entre razón y emociones, las discusiones orientadas a los efectos inmediatos de la violencia, etc. (Arendt, 2006; Sanmartín, 2002; Sosfky, 2006; Zúñiga, 2012). Todas estas terminan empujándonos a conclusiones que señalan a ciertos seres humanos que se suponen protagonizan la violencia, como carentes de cultura o bien, a la cultura como opuesta a la naturaleza humana; caracteriza a los seres humanos como irracionales o como altamente emocionales -como si esto fuera algo negativo-; la violencia como propia de personas malas, individual, una reacción incomprensible

2 Por ejemplo, en un deportista que busca ganar el primer lugar en una competencia, aun cuando se encuentra lesionado, vemos en su cuerpo la supuesta activación de una agresividad, la cual es empleada en algunas ocasiones como evidencia de que es la agresividad lo que mueve al ser humano hacia adelante. 
de un individuo hacia un grupo social, etc.; como se evidenciará en el acápite en que se aborda la comprensión de la violencia en el CFJZ.

Quizá una de las mejores formas de concebir la violencia fuera de estos esquemas más tradicionales, al menos para quienes buscamos realizar un acercamiento científico al tema, es considerarla como una propiedad emergente. ¿Qué quiere decir esto? que no podemos comprender la violencia como una cosa en sí misma, como una serie de elementos que le son propios y constitutivos, sino más bien como un proceso emergente de estados anteriores de organización de otras propiedades cuya interacción da como resultado eso que requerimos denominar violencia. Para Zúñiga (2012), por ejemplo, la violencia surge del conjunto de relaciones de un grupo humano. Esto da miras a posicionar un elemento fundamental en la comprensión de la violencia, como una propiedad que emerge de un proceso; de la sociabilidad, de los seres humanos.

Lo que mueve a los seres humanos es la necesidad de pertenecer, ser reconocido, formar parte de un grupo humano. Según Bauer (2013), un ser humano experimenta dolor cuando es menospreciado, rechazado, excluido socialmente, de igual forma que experimenta el dolor físico. Como cualquier otro organismo, requiere pertenecer a un entorno, que en el caso de los humanos no solo lo conforman los elementos bióticos y abióticos de la naturaleza externa que lo rodea, sino que también, y sobre todo, los procesos culturales de este, el tejido social que le da forma (Monsalve y Serrano, 2005; Zavala, 2012), a eso propio de un determinado grupo que denominamos cultura. El grupo de pertenencia es el entorno biosocial en el cual se mueve un ser humano (Monsalve y Serrano, 2005), es con y a partir de este que se definen todas las demás pertenencias en relación con la naturaleza que lo rodea y con los otros grupos, etc. (Maalouf, 2012). La sociabilidad, en los seres humanos, quiere decir que todo lo que hacemos es para, por y frente a otros. Partimos de que nuestra cultura es producto de estas relaciones que establecemos con los otros y de que no hay cultura fuera de esta relación (Carrithers, 1995).

Cuando decimos que la violencia es una propiedad emergente, producto de esta sociabilidad, queremos decir que la violencia es un proceso cultural; que existe gracias al conjunto de relaciones establecidas por los seres humanos entre sí, a la vez que hace posible dichas relaciones en el curso de movimiento que este tiene en el entorno biosocial en el cual se desarrolla. Todo lo que hacen los humanos está dirigido a la constitución del entorno en donde se desenvuelven, y este a la vez los constituye. Es decir, puede ser comprendido más allá de una lógica instrumental medio-fin, dentro de una lógica reproductiva en la cual este hacer está dirigido a la conservación de las condiciones del entorno que posibilitan la vida (Hinkelammert, 2005). Es en este movimiento que el ser humano, una persona-organismo (Ignold, 2008), adquiere sentido en un entorno particular; es decir, "experimenta un proceso de crecimiento y desarrollo en un contexto y [...] contribuye con su presencia y actividad al desarrollo de otros" (Ignold, 2008, p. 12).

De la relación entre violencia y sociabilidad se desprenden varios puntos importantes que serán abordados con la evidencia recolectada durante el trabajo de campo. En un primer momento se muestra que al no ser la violencia un proceso relacionado con la agresividad, sino con la cultura, no es un elemento exclusivo 
de personas impulsivas o incapaces de controlar sus instintos, sino un proceso del cual son participes diversos actores, y se manifiesta en la selectividad de usuarios reclutados para el sistema penitenciario y de las condiciones de vida a la que los exponen. En un segundo momento, la violencia funciona tanto para clasificar, e imponer a los otros una serie de atribuciones, como malos, impulsivos, etc., pero que en ese mismo proceso posibilita a las personas quienes clasifican reproducir formas de violencia y legitimarse, ocupando un lugar en el entorno en que se encuentran. Además, finalmente, cómo esas personas, consideradas como artífices de la violencia, pueden a su vez buscar un lugar legítimo que ocupar en el entorno carcelario, resaltando su condición de sobrevivientes.

\section{Violencia en los centros penales}

Si decimos que lo que mueve a los seres humanos es la necesidad de pertenecer ${ }^{3}$, ser reconocido, formar parte de un grupo humano y que la violencia es un proceso cultural emergente de las relaciones entre personas que buscan la constitución de un entorno y un movimiento en común con este, quiere decir, entonces, que las manifestaciones de violencia en un centro penal surgen del involucramiento mutuo de la totalidad de personas quienes interactúan en dicho centro, como grupo, que posibilitan la constitución de un entorno carcelario en el que cada uno de los actores ocupe un lugar y adquiera un sentido en la dinámica diaria ahí desarrollada.

Los centros penales son entornos en donde se coloca, en primer término en los organismos, la condición de "especialistas en fomentar y reproducir la ficcionalización de las personas" (Narciso, 2012, pp. 125-126), en otras palabras, en hacer que las personas "sean" de una determinada forma según los requerimientos de este entorno; un manejo de la fachada, esto mediante la demostración pública de ciertos valores, actitudes y comportamientos con miras a asegurar encajar en el entorno, y con esto la supervivencia física, psicológica y social (de Vigianni, 2012). Esto ha sido demostrado, inclusive, en entornos de prisión artificiales de corte experimental (Zimbardo, 2015).

En términos de Maalouf(2012) lo que determina la pertenencia de una persona a un grupo, su inclusión o exclusión, depende fundamentalmente de la influencia de los demás, agrega que:

3 Esta idea no es para nada nueva, ya Darwin (2009) había señalado la importancia de este aspecto en las formas de actuar de los seres humanos, pues para él los seres humanos poseían una "simpatía instintiva" que los llevaba a una constante búsqueda en la aprobación de sus iguales y acotaba que“ (...) por consiguiente, ejercen influencia muy grande en el (ser humano) y su conducta, los deseos, la aprobación o la censura de sus semejantes, ya se expresen por gestos, ya por palabras (Darwin, 2009, p.135). 
... todo ser humano ha de optar personalmente entre unos caminos por los que se lo empuja a ir y otros que le están vedados o sembrados de trampas; no es él desde el principio, no se limita a $<<$ tomar conciencia $>>$ de lo que es, sino que se hace lo que es; no se limita $\mathrm{a}<<$ tomar conciencia $>>$ de su identidad sino que la va adquiriendo paso a paso (p. 35).

\section{Origen sociodemográfico de la población privada de libertad}

Para comprender quiénes son las personas privadas de libertad y qué lugar ocupan en un contexto carcelario, es decir, cuál es el sentido que tienen en el desarrollo del funcionamiento diario del centro, se hace necesario considerar las características que anteceden a la posibilidad de hacerse "usuario" de dicha institución. Es decir, tener en cuenta que este tipo de instituciones están dispuestas contra los pobres y marginales del orden económico neoliberal (Wacquant, 2000), con miras a la neutralización física de los excedentes de los miembros desposeídos de grupos estigmatizados, la imposición de una disciplina de mano de obra desocializada y la reafirmación de la voluntad del Estado -penal- y las élites políticas de quienes deben ser considerados o no ciudadanos meritorios o desviados (Wacquant, 2012).

Según los informes penitenciarios del Poder Judicial de Costa Rica (MJP, 2014), el Programa de Atención Institucional está constituido por unas 13903 personas privadas de libertad, de las que el $85 \%$ son personas de nacionalidad costarricense. Del total de población privada de libertad, más del $50 \%$ tiene una condición educativa baja ${ }^{4}$. De los hombres y mujeres privados de libertad, el 58,8 \% son personas adultas jóvenes; es decir, entre los 18 y 35 años de edad -no incluye la población con sanciones penales juveniles-. La mayoría descuenta una sentencia por delitos contra la propiedad $(51,75 \%)$, seguidos de delitos contra la Ley de psicotrópicos $(18,54 \%)$, delitos contra la vida (16,54\%), y delitos sexuales $(6,80 \%)$.

Los centros penales están orientados al procesamiento de personas pobres y hay en estas instituciones una sobrerrepresentación de estos. Es sabido que los privados de libertad suelen ser personas que acumulan toda una serie de características socialmente desfavorables; lo que vuelve a estas manifestaciones de la violencia; la desigualdad y la exclusión social, económica y política; una primera condición de posibilidad de los centros penales como institución. Una primera legitimación de su dinámica institucional es la de procesar una categoría de personas consideradas socialmente como desviadas, y en la actualidad, estéticamente caracterizadas como "chatas"5.

4 Un 5,2 \% de la población es analfabeta, un $22 \%$ no finalizó la primaria, un $30 \%$ tiene grado de primaria completa, un $24,9 \%$ no ha terminado sus estudios de secundaria y solo un 4,7\% declaró tener estudios secundarios completos.

5 "Chata" es la forma en la que se autodenominan las personas que portan una cierta estética relacionada con el mundo musical 


\section{Condiciones de privación de libertad}

Esa violencia que legitima la dinámica institucional en los centros penales no se restringe a esa primera presión selectiva hacia ciertos organismos en vez de otros para hacerse usuarios de dichos centros. En un segundo momento de esa violencia, podríamos pensar en otros muchos actores (externos al centro) para cuya salud mental depende saber que las personas quienes han cometido un delito "reciben su merecido". Independientemente de que un centro penal sea visto como un medio de castigo o de rehabilitación, en términos prácticos, estos en nuestro país suelen someter innecesariamente a los privados de libertad a una serie de mortificaciones incompatibles con la condición de ser humano.

El hacinamiento y las condiciones de vida deplorables de la población consecuencias de infraestructura obsoleta -por no decir la institución misma- y de la falta de inversión en la construcción de lugares adecuados para el desarrollo de la vida humana de las personas en condición de encierro son dos manifestaciones claras de la violencia; las más alarmantes y explotadas por los medios de comunicación, a la vez que son las menos problematizadas como tal. De hecho, en informes específicos sobre el tema de violencia intracarcelaria en Costa Rica, ambos suelen ser referidos como "factores generadores de violencia" (Vargas, Montero y Fernández, 2013, p. 25). Cuando se habla de violencia carcelaria de forma explícita, en los medios de comunicación, en los informes oficiales de las instituciones, etc., generalmente se hace referencia a sus manifestaciones más físicas, agresivas o, en otros términos, evidentes (p.e cantidad de personas que son apuñaladas). Lo demás se convierte en meros elementos de contexto.

Según el Mecanismo Nacional de Prevención de la Tortura (MNPT), Costa Rica tiene una tasa de personas privadas de libertad muy por encima de otros países de América Latina, cuyo promedio es de 213 personas por cada cien mil habitantes, mientras que nuestro país es de 321 por cada cien mil. El promedio de hacinamiento era de 31,8 \% para el 2012 y de $41 \%$ para el 2014. Para el 2015, la proyección de hacinamiento era superior al $51 \%$, lo cual se considera una cifra récord. Las causas de estas situaciones de hacinamiento responden al crecimiento de la población penitenciaria debido a nuevas y más altas penas privativas de libertad, a la puesta en práctica de los tribunales de flagrancia en 2008, al aumento en el uso de procesos abreviados, al aumento de la prisión preventiva y a la subejecución en el presupuesto de construcción para nuevos espacios carcelarios (MNPT, 2015).

con el cual se identifican, generalmente el dancehall o el reggaetón. En el caso de los hombres, visten con ropa holgada, generalmente de colores llamativos, tenis deportivos de caña alta. El concepto se suele usar de forma peyorativa para quienes no son parte de esta grupalidad urbana, y se usa para designar tanto a los que portan esta estética original de los años 90 como para otras formas estéticas de vestir propias de los barrios urbano populares, generalmente ropa deportiva relacionada con deportes de velocidad, como las motocicletas, igualmente caracterizada por colores llamativos. 
Las consecuencias de esta situación de hacinamiento son claras, condiciones de vida deplorables para la población privada de libertad, para los oficiales de policía, y para los demás actores involucrados en el funcionamiento diario de un centro penal. Hay deterioro de las instalaciones físicas del centro, problemas de convivencia, recurrencia de lo que se ha llamado "violencia intracarcelaria", entre otras (Vargas, Montero y Fernández, 2013). Sin embargo, lo anterior no quiere decir que haya un mal funcionamiento de los centros penales, sino más bien responden al funcionamiento óptimo de la lógica que sostiene a esta institución, que tiene la generalización de la condición de "delincuente" a la totalidad de la persona (Foucault, 2009). Si se le quiere, pone el hecho de que la persona haya cometido un delito como elemento principal en la definición de la persona por sobre otros aspectos que la constituyen.

Cuando los medios de comunicación evidencian las condiciones de vida en las que se encuentran la población privada de libertad, aparece más como una verificación que como una denuncia o una crítica; efectivamente, cuando a un sujeto se le considera delincuente y no persona, se espera que se encuentre en tales condiciones. Es por ello que cuando sectores que buscan garantizar el respeto a los derechos humanos para dichas personas proponen alternativas que mitiguen estas situaciones, son acusados de favorecer a los "delincuentes" por encima de las "víctimas".

\section{Violencia en el Centro de Formación Juvenil Zurquí}

- El centro no es el encargado de definir si la persona es o no imputable, o si la intención era o no causar daño. El centro trabaja con personas que han sido señaladas como imputables. Ya hay una imputabilidad. Eso ya fue evaluado previo al ingreso (Comunicación personal, funcionaria del CFJZ, Nota diario de campo, 2015).

- Comenzamos a decir... Bueno si tenemos una persona adicta que entra a un centro de rehabilitación, ¿a qué entra? A trabajar la adicción, no. Raro sería que entre a un centro de rehabilitación y no se le trabaje la adicción. Entonces empezamos a mirar, ¿por qué entran los muchachos al Zurquí? Porque cometen un delito (Comunicación personal, funcionaria del CFJZ, Nota diario de campo, 2015).

Los dos extractos anteriores corresponden a las anotaciones de campo realizadas a partir de una charla dada por una funcionaria del área técnica del CFJZ a propósito de las generalidades por considerar en el abordaje de la violencia y el delito en este centro. En esta se definen dos elementos fundamentales de la función del centro penal; se trabaja con personas responsables de un delito y lo que caracteriza la relación de la institución con esa persona es precisamente el hecho de que lo haya cometido. Es a partir de esta certeza que se definen y legitiman tanto la función de profesional del área técnica en el centro así como la 
posición que ocupa la PJPL con respecto a dicho profesional; le concierne en este caso a este profesional la precisión del tema de la violencia.

La forma de comprensión de la violencia está dada por una articulación y distinción entre tres conceptos claves, manejados a nivel técnico para el abordaje de la población con la cual trabajan, estos conceptos son: agresividad, violencia y delito. La agresividad es entendida de dos formas; por un lado, hay una agresividad benigna que le es propia a todos los seres humanos la cual les posibilita a las personas protegerse del peligro o asirse de algún bien, como señala una de las profesionales del centro "todos vamos a tener un instinto más agresivo"; por otro lado, hay una forma de agresividad considerada como maligna, la cual se relaciona directamente con una intencionalidad de daño. De estos supuestos sobre la agresividad es que esta establece una relación con el tema de la violencia, en la que consideran que "hay agresión sin violencia, pero no existe violencia sin agresión”. Una vez hecha esta relación, se pasa, entonces, a considerar el aspecto del delito, como se muestra a continuación:

No podríamos decir que el delito es un delito no violento. El delito, aunque no se genera la violencia que se indica de forma más legal o más jurídica; como la violencia contra las personas, la violencia contra las cosas; en el delito [...] por sí mismo hay una intencionalidad de daño, entonces al haber una intencionalidad de daño, se convierte en una agresión maligna, y al tener daño a otros se convierte en violencia. Y como no hay violencia sin agresión entonces el delito lo vamos a entender como un acto violento (Comunicación personal, funcionaria del CFJZ, Nota diario de campo, 2015).

Es decir, en el centro se trabaja con el hecho de que las personas son responsables de un delito; y dentro del esquema anteriormente descrito, son protagonistas de violencia, una con un componente de agresividad maligna cuya característica es la intencionalidad de causar daño. Recordemos; no existe delito que no sea violento. A esto, se le unen otras formas de considerar a las personas privadas de libertad, como peligrosas, tendientes a la evasión, etc. Al final, la violencia se restringe a aquellas quienes tienen la intencionalidad de causar daño, lo cual puede excluir a todos aquellos actores que, aun cuando sus actos puedan ser considerados como agresivos, si su intencionalidad no es dañar, no podrían ser considerados como violencia. Los profesionales del área técnica y los oficiales estarían por fuera de ese protagonismo de violencia, pues actúan con miras a la consecución de un objetivo en común; la rehabilitación y educación de las PJPL que se encuentran en el centro. De hecho, al momento de preguntar a una de las funcionarias del centro acerca de que entienden ellos por violencia, su respuesta vadirigida a acciones o situaciones propias de la población. 
Violencia absolutamente toda, (...) desde el arremeter formalmente a alguien, con puño o con punzón o sexualmente, hasta lo que nosotros podríamos decir que son amenazas o son intimidación que suele pasar, como amarrar, amordazar, intimidar, cobrar, o someter a hacer algo que la persona no quiera hacer, como lavar ropa, como tener una experiencia sexual determinada o cercana, entonces cualquiera de esas para nosotros siguen siendo formas de violencia (Entrevista funcionaria CFJZ, octubre, 27, 2015).

Esta forma de concepción de la violencia es solo un ejemplo de cómo se lleva a cabo ese proceso mediante el cual las PJPL "pasan a formar parte de la categoría criminalizadora de "delincuentes juveniles"” (Unicef, 2001). Algunos autores le han llamado a esto etiquetaje, y lo han abordado como una forma de violencia simbólica (Venceslao, 2012), pero ciertamente puede ser considerado como parte del proceso de ficcionalización de la persona (Narciso, 2012) de parte de los profesionales que también deben mostrar una cierta regularidad en los valores, actitudes y comportamientos para mantenerse encajados en la dinámica social.

Durante mi interacción con estas personas profesionales en el centro, siempre se introducían de una forma muy comedida. Como profesionales del área técnica con sus conocimientos y experiencias, siempre bien presentados, excelentes y absolutamente conscientes en sus formas de trato, dispuestos para el trabajo de determinar, analizar, evidenciar y corregir en las personas privadas de libertad aquellos elementos de su proceso de desarrollo -denominados como factores de riesgo- que en algún momento determinado de sus vidas los llevaron a obrar de forma inadecuada o al menos de una no aceptada socialmente; o bien, encargados de la supervisión y corrección de los aspectos cotidianos de la convivencia de la población a lo interno del centro.

Tanto en mis notas de diario de campo como en las entrevistas a las PJPL que realicé, el profesional del área técnica es un actor poco presente en el entorno inmediato de la población, más allá de este rol formalmente definido desde la institución. Para la población privada de libertad, la interacción con estos es estrictamente formal, y se evidenciaba de esta forma en la mayoría de las entrevistas; como en el siguiente extracto de una de las entrevistas

(...) porque yo creo que el trabajo de ellos es solo estar allá arriba, ese es el trabajo de ellos, estar allá arriba, ellos trabajan allá arriba y, y cuando tienen que hablar 
algo con uno, diay yo pienso que la mayoría... bueno a mí todavía no me han llamado para cosas malas, pero yo pienso que si a uno lo llaman es porque pasó algo o porque tienen algo malo que decirle a uno (Jesús, PJPL, 17 junio, 2015).

\section{Oficiales de policía penitenciaria}

Cuando "la violencia" se define tanto como acto como a partir de la intencionalidad de daño, cabe la posibilidad de plantear una especie de gradualidad en la producción, reproducción o ejercicio de esta por parte de los diversos actores en el centro penal. Los profesionales del área técnica estarían muy lejos de una responsabilidad en su (re) producción, puesto que su función consiste más en la atención y en la toma de decisiones, donde media sobre todo la palabra y el razonamiento. Los oficiales de policía penitenciaria que tienen una interacción y roce más directo con la población, dentro de este esquema, podrían considerarse un poco más cerca en cuanto a responsabilidad por la violencia vivida en el centro penal, sin embargo, si se arguye que no hay una intencionalidad de daño, esta se restringiría a un "uso necesario de la fuerza".

Durante el trabajo de campo, los oficiales aparecían en el cumplimiento de un rol muy específico definido por la idea de seguridad o peligro. Ellos son los encargados de la supervisión, mantenimiento el orden, custodia, vigilancia, evitar fugas, asegurar cumplimiento de las reglas de convivencia, etc. Estos se presentan como personas duras, pero justas, siempre atendiendo a la labor, incomprendidos la mayoría de las veces, olvidados por el Estado, segregados de las otras policías, dispuestos a usar la fuerza solo en la medida de lo necesario. Igualmente, como fieles sensores de los valores que son buenos y aceptados socialmente, los cuales deben ser inculcados a la población.

El papel que ellos cumplen en el centro siempre está legitimado en función de la característica de peligro atribuida a la población; Para los oficiales, esta representa una amenaza y un peligro constante. La relación o interacción que establecen con ellos es en función a este precepto; no es algo que ellos puedan decidir, es simplemente así, una vez que los califican como "maleantes" o "delincuentes", deben actuar en consecuencia. De hecho, a pesar de que la mayor parte del tiempo permanecía cerca de las personas de las secciones preferenciales, la advertencia de los oficiales en aras de asegurar mi integridad siempre era la misma; "no podía fiarme de las personas de la sección, aun cuando parecieran tranquilas, pues todos estaban por delitos graves y en cualquier momento me podían provocar daño" (Nota diario de campo, mayo 17, 2015).

En los siguientes extractos del diario de campo se ejemplifican dos situaciones puntuales de esa definición de peligrosidad de la población por parte de los oficiales. La primera corresponde a una situación de conflicto presentada en la escuela del centro; y la segunda, a uno de los recorridos habituales a las unidades de convivencia (celdas) que realizaba durante mi trabajo de campo 
Estando ahí (en la escuela) de un pronto a otro se escuchó un desorden de sillas y el movimiento de los oficiales. ¡Bronca! ${ }^{6} \mathrm{O}$ como dicen ellos "se mosquió" oficiales agarraban a uno de los muchachos que (quería agredir) a otro de la sección contraria. "Un contraparte" o un "tronco". Yo entré al aula (...) para evitar cualquier inconveniente, pero me quedé de pie en la puerta. Hasta que una de las oficiales que estaba custodiando (...) en el aula (...) me solicitó que ingresara y que no me expusiera: "usted no tiene que exponerse. Uno de esos muchachos lo puede jalar y prensar del cuello. Y pide algo (se refiere a negociar como en los motines) y hay que ver que se hace. Usted no sabe del caso en la reforma donde murieron dos compañeros (oficiales)" (Nota diario de campo, marzo 3, 2015).

En una de las visitas de campo fui a la sección D3, en donde se encuentran personas adultas jóvenes privadas de libertad, uno de los jóvenes, "Thomas" extendió la mano para saludarme y solicitarme que le regalara un cigarro, de inmediato el oficial facilitador se me acercó y con un gesto me llamó un poco más lejos de la reja para decirme: "no se le acerque mucho a ese negro, es peligroso..." (Nota diario de campo, mayo 12, 2015).

El, oficial tiene sentido en la dinámica propia del contexto carcelario en cuanto se defina en oposición a la población y en un constante reforzamiento de su rol según las practicas relacionadas con esta. Su función es mantener la seguridad para lo cual deben superar en todo momento el ingenio de las PJPL. Como se muestra en el siguiente extracto:

Una funcionaria del área del Departamento Legal del Ministerio se encontraba realizando una visita al CFJZ, aparentemente estaba recopilando información sobre las condiciones de vida de la población, y reuniendo unos documentos (bitácoras oficialía de guardia) para su trabajo en el departamento. Estaba junto con el jefe de la policía de guardia y la secretaria de oficialía. El jefe estaba mostrándole algunas fotos de una de las estrategias que utilizan las personas para ingresar droga al centro (en una preparación de carne), lo que suscitó una conversación al respecto. En ese momento comenzaron a intercambiar opiniones sobre el trabajo de las personas por ocultar la droga, el cual parecía de admirar, sin embargo, de inmediato el jefe de la policía respondió que el admiraba más la labor del compañero que descubrió la droga en la carne. A lo que la funcionaría del Departamento Legal respondió: "para

6Riña o discusión fuerte.

7 Situación en la que hay gran confusión, ruido y griterío.

8 Contraparte y tronco son dos formas de referirse a otra persona como enemigo. 
ser oficial hay que ser peor delincuente que los delincuentes", lo decía en el sentido de tener la posibilidad de prever este tipo de acciones en las cuales se transporta la droga, a lo que el jefe de policía respondió de inmediato de forma afirmativa (Nota diario de campo, junio 9, 2015).

Tanto en el caso de los profesionales del área técnica como de los oficiales, estas formas de etiquetar o considerar a las PJPL son formas de violencia simbólica y tienen toda una serie de repercusiones no solo a nivel discursivo, sino también práctico (Venceslao, 2012). Por ejemplo, en situaciones de conflicto, siempre tiende a recaer sobre aspectos disposicionales del privado de libertad, ya sea que se responsabilice la impulsividad de la persona que no puede controlarse o la instrumentalidad que haga de la violencia o la manipulación para obtener algún beneficio; lo que termina por concretizarse en reportes de conducta, perdida de "beneficios" o de plazas laborales, aplicación de medidas disciplinarias, etc.

\section{Población joven privada de libertad}

Como se vio anteriormente, el centro penal pone en primer término ficcionalizar la persona quien se espera, en un contexto carcelario. Esto es cierto no solo para los privados de libertad, quienes tratan de ser algo que realmente no son (de Vigianni, 2012), sino que también para los profesionales del área técnica y oficiales de policía, quienes en lugar y tiempo deben mantenerse en su rol; y es desde el cumplimiento rígido de ese rol que se establece la posibilidad de relacionarse con otros actores; sobre todo en relación con la población privada de libertad.

A las PJPL les son atribuidas una serie de características por parte de los funcionarios como malcriados, sin límites irrespetuosos, destructores, agresivos, inmaduros, impredecibles, desconfiables; responsables a causa de su naturaleza destructiva de las malas condiciones de vida en las cuales se encuentran, entre otras. Como se señala en el siguiente extracto de entrevista: "puede(n) ser a veces más peligroso(s) que cualquier otro porque está(n) en una época de efervescencia, porque es... ¿verdad? impulsivo etcétera" (Entrevista a funcionaria, 2015). Esto los vuelve blanco de constantes amenazas de traslado a otro centro penal o bien, de pasarles un reporte el cual afectaría sus expedientes o podían costarles las plazas laborales u otros "beneficios". Durante el trabajo de campo fue recurrente la advertencia de que en el centro "solo se queda población de bajo perfil, es decir con condenas bajas que no tengan causas como adultos, que tengan buen comportamiento y sin reportes de mala conducta. Todo lo que no nos funcione se va..." (Nota diario de campo, enero 16, 2015). Sin embargo, lo principal en la población es presentarse como supervivientes. Si lo que definía o legitimaba la posición de los profesionales del área técnica era la educación y la transformación, a los oficiales la seguridad y la peligrosidad, a las personas jóvenes privadas de libertad lo hace la supervivencia. La palabra violencia no es algo que circule con frecuencia entre la población, durante el trabajo de campo solo en una ocasión fue utilizada por uno de los muchachos del centro, para referirse a la forma en la cual solucionar los conflictos, "diay como, como diay, muchas veces también se intentó hablar, 
muchas veces se intentó hablar, pero diay, casi siempre diay se nos sale la violencia la verdá (sic), (...) diay la violencia porque diay, es lo que queda siempre" (Álvaro, PJPL, julio 1, 2015).

Hay dos ejemplos clave para entender este énfasis en la supervivencia hecha por la población. La primera tiene que ver con la fabricación de armas hechizas. Este es uno de los grandes problemas a los que se enfrentan cotidianamente los oficiales de seguridad; por un lado, porque, junto al decomiso de drogas y otros objetos prohibidos, se convierte en una rutina diaria para mantener la seguridad; por otro, por la gran cantidad de incidentes de agresiones o autoagresiones que son producto de la presencia de armas hechizas. Para la población, es cuestión de asegurar recursos, así como la integridad física frente a otros privados de libertad. Con respecto a lo primero, uno de los entrevistados señalaba lo siguiente:

(Hay tres tipos de termitas ${ }^{9}$ ) Las de hierro, los que cortan y los que desbaratan paredes para sacar las bichas ${ }^{10}$. (...) Diay mae, es, ¿cómo le explico?, es su defensa personal, muchas veces (de) eso puede (depender) su vida (sic), hay muchas requisas... muchas, muchas varas se caen y hay mucha gente que vive de eso, de cortar (...) la vara, agarran y hacen bichas y las venden y así (Gordo Jack, PJPL, agosto $24,2015)$.

De igual form, es por las armas hechizas que las personas pueden asegurarse que llegado el momento de enfrentarse a alguien, van a poder asegurar su supervivencia física:

Usted sabe que también en el momento en el que usted le lleguen (la bicha) es su modo de defensa, aunque sea, aunque sea uno se arranca, ¿entiende?, entonces es, es como, ¿cómo le explico? Como... supervivencia, es como los animales (...), ellos tienen que defenderse (...), por más impotentes que (sean) siempre tienen que buscar una forma de defenderse (sic), ¿entiende? Es decir, corre el riesgo de que lo apuñalen (Gordo Jack, PJPL, agosto 24, 2015).

Para las personas privadas de libertad, tanto el entorno como quienes son parte de este también representan amenaza y peligro. La fabricación y la portación de armas es una forma de asegurar su integridad, también lo son la desconfianza y la agresión. Sobre la desconfianza, tanto en las observaciones como en las entrevistas, quedaba algo muy claro, el que se confía del entorno puede resultar agredido y el que muestra confianza en otra persona dentro de prisión puede terminar traicionado. Esto último queda claro en el siguiente extracto de una de las entrevistas realizadas a la población:

9 Termita es la persona que se dedica a extraer de la planta física del centro, objetos que puedan ser utilizados como materia prima para la fabricación de armas hechizas.

10 Es una de las formas en las que se refieren a las armas hechizas. 
Al chile mentile (sic), llevármelo viajado y montarlo en otra psicológica, ¿por qué?, porque (es) el vivir de uno, del día a día estando preso y de todo... diay solo es la forma de uno del preso (sic) y de todo y la sociedad no tiene que saberlo, no tiene que saberlo nada (sic) porque es la forma de convivir de nosotros. Por medio de algo que yo le diga a usted y de todo y la paca ${ }^{11}$ se da cuenta y de todo son varas que le van a servir (...) para encontrar galetas ${ }^{12}$ (sic) y, y varas así, ¿me entiende? Entoes (sic) uno solo se estaría perjudicando (Gordo Jack, agosto 24, 2015).

En cuanto a la agresión, y esto es el segundo ejemplo sobre el énfasis en la supervivencia, es fundamental en dos sentidos: asegurar el respeto y verificar la virilidad. Aquí la agresión cumple su función comunicativa (Bauer, 2013), decirle a quienes me rodean que no se está dispuesto a soportar ser considerado como inferior o excluido, o en términos de la población "no hay que dejarse agarrar como un playo ${ }^{13}$ ", como vemos en los siguientes extractos de entrevista:

(...) unas pintillas llegaron a apestarme $\mathrm{e}^{14} \mathrm{y}$ los agarré a pichazos ${ }^{15} \mathrm{y}$ la vara, pa que los maes vieran que diay sí, que ya uno, uno no se les va a dejar ni nada, y con eso me $\operatorname{soltaron}^{16}(\ldots)$ es muy importante porque si no le van a ver cara de playo, $(. .$.$) uno no$ tiene que dejar que nadie le vea la cara de playo nada más (Jesús, PJPL, 17 junio, 2015).

\section{¿El centro penal es violento en sí mismo?}

Como se dijo al inicio de este texto, la posible interpretación de esta expresión; la violencia como producto de las condiciones de vida deplorables a las que se someten a las personas privadas de libertad; resulta insuficiente y parcial para comprender el fenómeno. Deja de lado la importancia que tiene la necesidad gregaria de los seres humanos de formar parte en todo momento de los entornos en los que se desenvuelven (Bauer, 2013), de los cuales, las relaciones entre personas son un elemento constitutivo fundamental (Carrithers, 1995). Se deja de lado, por tanto, que las personas quienes son parte de los diversos sectores del centro penal profesionales del área técnica, oficiales de policía o privados de libertad, en tanto permanecen en mayor o menor tiempo en este lugar, requieren ajustarse a la lógica de dicho sector según les permita ocupar un lugar legítimo en la institución (Padrón, 2006).

11 Es la forma de referirse a la policía y a los oficiales penitenciarios.

12 Caletas, encaletar, tener encaletado. Se refiere a los lugares en los que se tienen objetos escondidos o guardados.

13 Homosexual.

14 Molestar.

15 Golpes.

16 Dejar tranquilo. 
Como se dijo anteriormente ficcionalizar es lo que fomenta y reproduce en primer término el centro penal (Narciso, 2012), lo que quiere decir que exige en todo momento de la persona una presentación, una fachada rígida y absoluta de lo que se supone debe ser (de Viggiani, 2012; Milchalski, 2015). La ejecución permanente, si se lo quiere, de un rol específico según la posición que el entorno posibilita ocupar en el centro, el cual se mencionó es producido por las personas -los organismos- mismos, conlleva que la gente pueda realizar "cosas horribles" cuando son muy rígidos los límites del papel que se supone debe representar (Zimbardo, 2015, pp. 296-297). Esto nos las hace especialmente malas o perversas, tampoco las exime de responsabilidad, solo las hace parte de un entono particularmente violento.

La mayoría de trabajos que abordan el tema de la violencia (intra) carcelaria suelen olvidarse u omiten intencionalmente esos demás actores que contribuyen con sus prácticas y necesidades a la constitución del entorno en el que se desenvuelven, además de considerar manifestaciones particulares de violencia como meros elementos de contexto, como caldo de cultivo para las acciones de los privados de libertad (de Viggiani, 2012; Milchalski, 2015; Vargas, Montero y Fernández, 2013). La violencia suele referirse casi que exclusivamente a lo que sucede entre las personas privadas de libertad, a la disputa por recursos, al monopolio en el uso de la violencia como elemento simbólico o de organización de las relaciones sociales, a reacciones ante las malas condiciones de vida, a la socialización hipermasculinizada y basada en el estatus entre varones privados de libertad, etc. (Crespo, 2009; de Viggiani, 2012; Milchalski, 2015; Vargas, Montero y Fernández, 2013).

Si queremos entender la violencia en los centros penales y qué significa que la cárcel sea violenta en sí misma, hay que comenzar por aceptar que esta se genera en primera instancia fuera de estas instituciones; que esta emerge de la forma de relacionarse de las personas de los diferentes sectores, tanto de la sociedad en general como de la institución.

En primer lugar, la desigualdad y la exclusión social, como manifestación de violencia, llevan a una "exclusividad" de personas de los sectores menos favorecidos para engrosar las filas de la cara más obvia del Estado Penal (Wacquant, 2012), proceso del cual todos en mayor o menor medida somos afectados, cuando nos conmueve el dolor que nos produce la exclusión de estas personas (Bauer, 2013), como ofendidos cuando la exclusión nos toca directamente a nosotros mismos o como ofensores cuando nuestros estilos de vida llevan necesariamente a la exclusión de otros sectores. Esto legitima nuestra forma de guiarnos política, económica y culturalmente en las sociedades modernas, tendientes, como señala Bauman (2015), a la producción de humanos residuales. Esto nos permite ocupar un lugar dentro de su lógica.

En segundo lugar, cuando celebramos, fomentamos, callamos o instrumentalizamos las condiciones de vida deplorables en las que se encuentran las personas privadas de libertad en los diversos centros penales del país (de Barcellos, 2010). Cuando estas condiciones no son consideradas en sí mismas como una manifestación de violencia, que tienen claramente a la sociedad en general como ofensora, se legitima el trato cruel que la institución representa para la persona. En cierta formam la sociedad le revoca el derecho y la dignidad a la persona, la persona privada de libertad está siendo considerada a partir de la 
comisión de ciertos delitos o la presunción de haberlos cometido por lo que “(...) no tiene dignidad y, por tanto, en realidad no tiene derecho a ser tratado con dignidad (...) (sus derechos) no son realmente derechos per se y el debate sobre los mismos se limita al espacio de la benevolencia" (de Barcellos, 2010, p.16).

En tercer lugar, como se vio en el caso del CFJZ, que la naturaleza gregaria de los seres humanos quienes interactúan y se relacionan diariamente en ella requiere para su supervivencia, tanto fisiológica como social, mostrar una cierta regularidad en los valores, actitudes y comportamientos para encajar en el entorno carcelario; donde cada uno de los actores demuestra un rol rígido y generalmente polarizado con respecto a los otros, sobre todo a las PJPL, asignándole a estos últimos la responsabilidad sobre las manifestaciones de violencia, la peligrosidad y la amenaza; a la vez que estos se valen de esos elementos para justificar sus agresiones, la portación y fabricación de armas las cualesevocan la necesidad de supervivencia.

A modo de cierre, que la cárcel sea violenta en sí misma significa que sin violencia no hay centro penal; sin la desigualdad y la exclusión a lo externo del sistema penal no puede haber cada vez más personas que sean catalogadas como delincuentes y privadas de libertad en condiciones inadecuadas. De igual forma, si ese proceso no se da a lo interno del centro, no se puede considerar a las personas jóvenes privadas de libertad como peligrosas y amenazantes, para legitimar las acciones de los cuerpos de seguridad, o como personas violentas, y la labor de los profesionales en el área técnica, o en general de toda una serie de actores que se relacionan con ellos en calidad de educadores; o bien que se acepten las prácticas de las PJPL como estrategias para la supervivencia.

\section{Referencias bibliográficas}

Alpízar, W. y Rodríguez, C. (2004). Análisis de la autoestima de adolescentes internados bajo medida tutelar en el Centro de Formación Juvenil Zurquí (Tesis de Licenciatura inédita). Universidad de Costa Rica, San José, Costa Rica.

Arendt, H. (2006). Sobre la violencia. Madrid: Alianza Editorial.

Arguedas, J. (1999). Delincuencia juvenil: Sobre mitos y realidades (Tesis de licenciatura inédita). Universidad de Costa Rica, San José, Costa Rica.

Baños, A. (2005). Antropología de la violencia. Estudios de antropología biológica, 12, 41-63.

Bauer, J. (2013). La violencia cotidiana y global. Una reflexión sobre sus causas. España: Plataforma Actual.

Bauman, Z. (2015). Vidas desperdiciadas. La modernidad y sus parias. España: Paidós.

Blair, E. (2004). Mucha sangre y poco sentido: la masacre, por un análisis antropológico de la violencia. Boletín de antropología, 18; 165-184.

Bustos, A. (2013). Vivencias de adolescentes asociadas al delito de Robo Agravado. Estudio de casos en privados de libertad del Centro de Formación Juvenil Zurquí, Heredia (Tesis de licenciatura inédita). Universidad de Costa Rica, Guanacaste, Costa Rica. 
Calderón, R. (2012). Delito y cambio social en Costa Rica. San José: FLACSO.

Carrithers, M. (1995). ¿Por qué los humanos tenemos cultura? Una aproximación a la antropología y la diversidad social. España: Alianza Editorial.

Cerbino, M. (2011). Jóvenes víctimas de violencias, caras tatuadas y borramientos. Perfiles Latinoamericanos, 19(38), 9-38.

Chaves, J. (2011). Entre la violencia sobre el cuerpo y la violencia incorporada. Hacia la promoción de la salud, 16, 162-172.

Corrales, M. y León, S. (2007). Aplicación de la Ley de Ejecución de las Sanciones Penales Juveniles en relación con los derechos fundamentales de los jóvenes privados de libertad en centro especializado de internamiento (Tesis de licenciatura inédita). Universidad de Costa Rica, San José, Costa Rica.

Crespo, F. (2009). Cárceles, subcultura y violencia entre internos. Revista CENIPEC, 28, 123-150.

Darwin, C. (2009). El origen del hombre (Vol. 1). España: EDIMAT.

de Barcellos, A. P. (2010). Violencia urbana, condiciones carcelarias y dignidad humana. SELA (Seminario en Latinoamérica de Teoría Constitucional y Política) Paper 88. Recuperado de http://digitalcommons.law.yale.edu/yls_sela/88/

de Vigianni, N. (2012). Trying to be Something are you not: Masculine Performance within a Prisión Setting. Men and Masculinities, 15(3), 271-291.

Foucault, M. (2009). Vigilar y castigar. Nacimiento de la prisión. México D.F.: Siglo XXI.

Fuentes, G. (1998). Derechos laborales: Un reto para la población penitenciaria (Tesis de licenciatura inédita). Universidad de Costa Rica, San José, Costa Rica.

Hinkelammert, F. (2005). El sujeto y la Ley. El retorno del sujeto reprimido. Costa Rica: EUNA.

Ignold, T. (2008). Tres en uno: Cómo disolver las distinciones entre mente, cuerpo y cultura. En T. Sánchez (ed.), Tecnogénesis: La construcción técnica de las ecologías humanas (pp. 1-34). Madrid: AIBR.

Maalouf, A. ([1999] 2012). Identidades asesinas. España: Alianza Editorial.

Michalski, J. H. (2015). Status Hierarchies and Hegemonic Masculinity: A General Theory of Prison Violence. British Journal of Criminology, 14, doi: 10.1093/bjc/azv098

Ministerio de Justicia y Paz (MJP). (2014). Informe de población penitenciaria Poder Judicial, II Trimestre 2014. Recuperado de http://www.mjp.go.cr/Downloads/Estadistica/II\%20TRIMESTRE\%20 2014.pdf

Mecanismo Nacional de Prevención de la Tortura (MNPT). (2015). Informe anual de labores 2014. Recuperado de http://www.dhr.go.cr/prevencion_de_la_tortura/mecanismo_nacional_prevencion/09_informe_anual_labores_2014.html

Monsalve, T., y Serrano, C. (2005). La síntesis biosocial: Una propuesta teórica en antropología biológica. Boletín de Antropología Americana, 41, 5-16.

Narciso, L (2012). Emergentes identitarios en cárceles de Santa Fe, Argentina. Avá, 20, 113-141.

Padrón, M. (2006). La violencia carcelaria. Psicología, 24, 97-114.

Ramírez, B. (2010). Causas y motivaciones que según mujeres y hombres menores de edad les llevaron a delinquir y cumplir actualmente una sentencia en el Centro de Formación Juvenil Zurquí en Costa Rica (Tesis de licenciatura inédita). Universidad Nacional, Heredia, Costa Rica. 
Rojas, A. (2013). Análisis sobre el cumplimiento de la Ley de Ejecución de las Sanciones Penales Juveniles en relación con el principio de la formación integral del menor en el Centro de Formación Penal Juvenil Zurquí (Tesis de licenciatura inédita). Universidad de Costa Rica, San José, Costa Rica.

Rojas, L., y Hernández, H. (2011). Análisis del discurso resocializador de la pena privativa de libertad: Centro de atención institucional Cartago (Tesis de licenciatura inédita). Universidad de Costa Rica, San José, Costa Rica.

Sanmartín, J. (2002). La mente de los violentos. España: Ariel.

Sosfky, W. (2006). Tratado sobre la violencia. España: ABADA editores.

Unicef. (2001). En busca de oportunidades para adolescentes y jóvenes infractores: una propuesta de intervención. San José: Unicef.

Vargas, E., Montero, P., y Fernández, L. (2013). Las manifestaciones de la violencia intracarcelaria en los centros penitenciarios juveniles. San José: Mecanismo Nacional de Prevención de la Tortura (MNP).

Venceslao, M. (2012). Pedagogía correccional. Estudio antropológico sobre un Centro Educativo de Justica Juvenil (Tesis de doctorado inédita). Universitat de Barcelona, Barcelona, España.

Vul, M. (2009). Adolescencia y violencia: homicidio en un colegio público, una lectura desde el psicoanálisis (Tesis de maestría inédita). Universidad de Costa Rica, San José, Costa Rica.

Wacquant, L. (2000). Las cárceles de la miseria. Argentina: Manantial.

Wacquant, L. (2012). Castigar a los pobres. El gobierno neoliberal de la inseguridad social. España: Gedisa Editorial.

Zavala, J. (2012). Ontogenia y teoría biocultural. Bases para el estudio de la persona a partir del desarrollo infantil. México: CopIT-arXives.

Zimbardo, P. (2015). El efecto Lucifer. El porqué de la maldad. España: Paidós.

Žižek, S. (2009). Sobre la violencia: seis reflexiones marginales. España: Paidós.

Zúñiga, M. (2012). Violencia y sociedad en El Salvador (una reflexión categorial). Manuscrito inédito (Facilitado por el autor). 